Front versus rear side light-ion acceleration from high-intensity laser-solid interactions

This article has been downloaded from IOPscience. Please scroll down to see the full text article.

2011 Plasma Phys. Control. Fusion 53014011

(http://iopscience.iop.org/0741-3335/53/1/014011)

View the table of contents for this issue, or go to the journal homepage for more

Download details:

IP Address: 141.211.173.82

The article was downloaded on 06/04/2012 at 16:58

Please note that terms and conditions apply. 


\title{
Front versus rear side light-ion acceleration from high-intensity laser-solid interactions
}

\author{
L Willingale ${ }^{1}$, G M Petrov ${ }^{2}$, A Maksimchuk ${ }^{1}$, J Davis $^{2}$, R R Freeman ${ }^{3}$, \\ T Matsuoka $^{1}$, C D Murphy ${ }^{3}$, V M Ovchinnikov ${ }^{3}$, L Van Woerkom ${ }^{3}$ and \\ K Krushelnick ${ }^{1}$ \\ ${ }^{1}$ Center for Ultrafast Optical Science, University of Michigan, 2200 Bonisteel Boulevard, \\ Ann Arbor, MI 48109, USA \\ 2 Naval Research Laboratory, Plasma Physics Division, 4555 Overlook Avenue SW, Washington, \\ DC 20375, USA \\ ${ }^{3}$ Department of Physics, The Ohio State University, Columbus, OH 43210, USA \\ E-mail: wlouise@umich.edu
}

Received 21 October 2009, in final form 8 April 2010

Published 16 December 2010

Online at stacks.iop.org/PPCF/53/014011

\begin{abstract}
The source of ions accelerated from high-intensity laser interactions with thin foil targets is investigated by coating a deuterated plastic layer either on the front, rear or both surfaces of thin foil targets. The originating surface of the deuterons is therefore known and this method is used to assess the relative source contributions and maximum energies using a Thomson parabola spectrometer to obtain high-resolution light-ion spectra. Under these experimental conditions, laser intensity of $(0.5-2.5) \times 10^{19} \mathrm{~W} \mathrm{~cm}^{-2}$, pulse duration of $400 \mathrm{fs}$ and target thickness of $6-13 \mu \mathrm{m}$, deuterons originating from the front surface can gain comparable maximum energies as those from the rear surface and spectra from either side can deviate from Maxwellian. Two-dimensional particle-in-cell simulations model the acceleration and show that any presence of a proton rich contamination layer over the surface is detrimental to the deuteron acceleration from the rear surface, whereas it is likely to be less influential on the front side acceleration mechanism.
\end{abstract}

(Some figures in this article are in colour only in the electronic version)

\section{Introduction}

Ion acceleration from laser plasma interactions [1-3] is an active area of research with possible applications in hadron therapy [4-6], proton radiography [7] or as an energy source to start the burn in the fast ignition concept for inertial confinement fusion $[8,9]$. These ion beams possess desirable qualities such as small effective source size, high luminosity and small emittance. The target normal sheath acceleration (TNSA) mechanism [10] is discussed as the primary 
mechanism in many experiments measuring proton and ion beams in the rear target normal direction. The interaction of the laser with the front surface of the target generates a hot electron beam, which propagates through the target and moves out into the vacuum generating a strong space-charge sheath field. The rear surface becomes ionized and the protons and ions are accelerated from the rear surface in the target normal direction. Evidence for this TNSA mechanism was shown using a wedged target [3].

However, not all the ions accelerated in the target normal rear direction necessarily originate from the rear surface [11]. The laser interaction at the front surface provides at least two other possible ion acceleration mechanisms: skin-layer ponderomotive acceleration [12] or shock acceleration $[2,13,14]$. The ponderomotive force of the laser exerts a pressure on the target electrons driving them away from regions of highest intensity into the target. The relatively heavy ions are unaffected by the laser fields directly, but respond to the electric field due to the electron displacement. If the ponderomotive force is large enough, high Mach number electrostatic shocks can be driven into the target, which are also capable of accelerating ions [2,14]. Most experiments claim one of these acceleration mechanisms is dominant for a particular case; however, they can be difficult to distinguish experimentally [15].

Theoretical studies show that as laser intensities are increased, radiation pressure acceleration (RPA) [16] overtakes the sheath acceleration as the primary ion acceleration mechanism. Schemes such as RPA have the potential to produce quasi-monoenergetic ion beams with much higher energies than previously achieved experimentally (into the gigaelectronvolts range), which are desirable improvements. The laser piston regime, where laser pressure is dominant, was proposed by Esirkepov et al [16] and required a $I \sim 10^{21} \mathrm{~W} \mathrm{~cm}^{-2}$ linearly polarized pulse. Intensity requirements can be reduced by using a circularly polarized pulse to $>10^{20} \mathrm{~W} \mathrm{~cm}^{-2}$ for RPA [17] with optimum parameters being determined by a light-sail model [18] or via a combination of RPA and a directed Coulomb explosion [19]. Recent experimental results have shown that RPA can play a part in ion acceleration at much lower intensities $\left(\sim 5 \times 10^{19} \mathrm{~W} \mathrm{~cm}^{-2}\right)$ [20], which are currently routinely available for ion acceleration experiments. Therefore, interactions at even modest intensities can potentially shed light on future scalings for these models. It is worth noting that these schemes are based on the use of very thin foils for which the front and rear surfaces of the target are less well defined.

An extensive comparison has been performed by Fuchs et al who use boron activation to compare front to rear side acceleration mechanisms [21,22]. Either the front or rear surface of the target was coated with a thin layer of deuterated plastic, then either ${ }^{10} \mathrm{~B}(\mathrm{~d}, \mathrm{n}){ }^{11} \mathrm{C}$ or ${ }^{11} \mathrm{~B}(\mathrm{p}, \mathrm{n}){ }^{11} \mathrm{C}$ reactions are used to get a total yield for the whole proton or deuteron beam accelerated in the rear direction. A Maxwellian energy spectrum with a high-energy cut-off was assumed for the ions to analyze the activation data. This is a similar technique to that described in [2].

Presented here is a preliminary investigation comparing the front and rear light-ion acceleration mechanism. High-resolution light-ion spectra are measured from targets where a deuterated coating is placed on either the front, rear or both surfaces of the target, meaning the origin of the high-energy deuterons accelerated in the interaction is known. High-resolution ion spectra are measured in the target normal direction using a Thomson parabola spectrometer and the laser beam pointing is stable to within a focal spot diameter on the target, so that the maximum ion energies are observed in this direction. It is possible that more complicated beam structures could be present [1], but the maximum energy protons are still expected to be observed on the target normal axis. Deuterons originating from the front surface can be accelerated in the target normal rear direction to similar maximum energies and flux, as those originating on the rear surface for our experimental conditions. Two-dimensional 
particle-in-cell (PIC) simulations are used to model the front and rear ion acceleration. From these simulations it is clear that the existence of the proton rich contamination layer is detrimental to the rear-surface acceleration of deuterons. The front surface acceleration of deuterons is likely to be less influenced by the contamination layer due to pre-plasma formation. This experiment is motivated by the desire to accelerate a high-energy deuteron beam in order to generate a beam-target neutron beam using the $d(d, n)-{ }^{3} \mathrm{He}$ reaction with neutron energies upshifted from the center-of-mass energy of $2.45 \mathrm{MeV}$ [23-25].

\section{Experimental set-up}

The experiments were performed using the T-cubed laser at the University of Michigan, which is a CPA hybrid $\mathrm{Ti}$ : sapphire/Nd: phosphate glass system with a central wavelength of $1.053 \mu \mathrm{m}$. The on-target energy was up to $\mathcal{E}_{\mathrm{L}}=6 \mathrm{~J}$ in a $\tau_{\mathrm{FWHM}}=400 \mathrm{fs}$ full-width half-maximum (FWHM) pulse length. An $f / 2$ off-axis parabola focused the pulse to a $5 \mu \mathrm{m}$ diameter FWHM spot (containing 50\% of the energy) giving average vacuum intensities of up to $I_{0}=2.5 \times 10^{19} \mathrm{~W} \mathrm{~cm}^{-2}$ (normalized vector potential, $a_{0} \approx 4.5$ ) and is p-polarized on the target. The nanosecond energy contrast due to amplified spontaneous emission (ASE) was measured to be $\sim 5 \times 10^{-6}$ with a high-speed ( 1 ns rise time) Si detector (DET 10A, Thorlabs) and $1 \mathrm{GHz}$ bandwidth, $10 \mathrm{Gs} \mathrm{s}^{-1}$ sampling rate scope (Lecroy $104 \mathrm{MXi}$ ). This corresponds to an intensity contrast ratio of $2.5 \times 10^{8}$ or a pre-pulse intensity of $1 \times 10^{11} \mathrm{~W} \mathrm{~cm}^{-2}$. Ion acceleration can be sensitive to the ASE levels in at least three different ways. Firstly, the formation of a pre-plasma before the main pulse arrives can change the absorption of the laser energy into hot electrons [26-28]. Secondly a shock breakout at the rear surface can increase the density scale length on the rear surface, reducing the effectiveness of the TNSA mechanism [27, 29]. Thirdly, the local target normal direction on the rear of the target can be changed steering the beam away from the original normal direction [30].

The targets were $6 \mu \mathrm{m}$ thick aluminum or $13 \mu \mathrm{m}$ thick Mylar foils. A solution of deuterated polystyrene, $\mathrm{CD}$, dissolved in toluene was used to deposit a layer of $\mathrm{CD}$ on either the front, back or both target surfaces. The toluene evaporated to leave a film of $\sim 1 \mu \mathrm{m}$ thickness on the foil surface. It is expected that the usual hydrocarbon/water contaminant layer typically present on the foil also forms on top of this CD layer. This contaminant layer, having an estimated thickness of a few nanometers, can impede the acceleration of heavier ions, including deuterons.

The target was positioned at an angle of $22.5^{\circ}$ with respect to the incident p-polarized laser beam. A Thomson parabola spectrometer measured the ion spectra in the rear-surface target normal direction. A micro-channel plate (MCP) coupled to a phosphor screen was used to detect the ions and the light emitted from the phosphor screen was imaged onto a CCD camera. The response of the MCP and phosphor screen to the protons was calibrated using CR39 using a similar method described by Harres et al [31] to find the energy dependent scaling factor. The energy loss for both protons and deuterons with respect to ion velocity is approximately the same and therefore the scaling factor for the deuteron response can be calculated. The deflection of the ions in the Thomson parabola spectrometer is determined by the charge to mass ratio $(Z / A)$ of the ions. Deuterons, $\mathrm{d}^{+}$, have a $Z / A=1 / 2$ which is identical to fully ionized carbon, $\mathrm{C}^{6+}$ and fully ionized oxygen, $\mathrm{O}^{8+}$. In order to distinguish deuterons from carbon and oxygen ions, shots from coated and uncoated targets were compared. Shots onto uncoated targets produce typical ion spectra of protons, carbon up to $\mathrm{C}^{4+}$ and small numbers of low charge state oxygen ions. Therefore, when a signal is observed on the $Z / A=1 / 2$ parabola from a deuterated target, it can confidently be identified as deuterons. Angular divergence measurements were made of the proton beam from an uncoated target using a 
filter stack in front of the CR39 detector to get the full divergence angle for different proton energies.

\section{PIC simulations}

A series of simulations are performed to match the conditions of the experiments. The twodimensional, fully relativistic, electromagnetic PIC model developed in [24, 32] is used. The laser radiation, which propagates in the positive $x$-direction, is linearly polarized in the $y$-direction and normally incident on the target. The parameters of the laser beam used in the simulations are peak laser intensity $I_{0}=(0.5-3) \times 10^{19} \mathrm{~W} \mathrm{~cm}^{-2}$, pulse duration $\tau_{\mathrm{FWHM}}=400 \mathrm{fs}$ (full duration at the base $\tau_{0}=2 \tau_{\mathrm{FWHM}}=800 \mathrm{fs}$ ), focal spot $D_{\mathrm{FWHM}}=5 \mu \mathrm{m}$ (spot radius at $1 / e$ level $R_{0}=3 \mu \mathrm{m}$ ), wavelength $1 \mu \mathrm{m}$. The range of peak intensities has been chosen to roughly cover the range of laser intensities used in the experiments. The simulation box is a rectangle with dimensions $\left\{L_{x} \times L_{y}\right\}=30 \times 20 \mu \mathrm{m}^{2}$ divided by 1.5 million cells with size $\{\Delta x \times \Delta y\}=20 \times 20 \mathrm{~nm}^{2}$ each. The total number of particles used in the simulations is $25 \times 10^{6}$ with $\sim 70$ electrons per cell and $\sim 20$ ions per cell. The simulation time for all runs is 1 ps. The particles and electromagnetic field components are advanced with a time step $\Delta t=\frac{1}{4}(\Delta x / c) \cong 16.7$ ( $c$ is the speed of light), which corresponds to 200 steps per laser cycle.

The targets are rectangular and extend $18 \mu \mathrm{m}$ in the $y$-direction. A pre-plasma is assumed to have formed by the ASE pedestal of the laser pulse and is modeled by having a profile which falls off exponentially from the front surface of the target with a characteristic scale length $L_{\mathrm{p}}=\left[\partial \ln \left(n_{e}\right) / \partial x\right]^{-1}=0.2 \mu \mathrm{m}$ (at $1 / e$ level) and a total of $3 \mu \mathrm{m}$ of pre-plasma. This pre-plasma was chosen to maximize the absorption of laser energy and ion acceleration (i.e. a best case scenario) and is in fairly good agreement with hydrodynamic simulations performed under similar conditions [30]. There is a $5 \mu \mathrm{m}$ vacuum region between the pre-plasma and the edge of the simulation domain. The flat top part of the target is a total of $7 \mu \mathrm{m}$ long and has an electron density of $\sim 200 n_{\mathrm{c}}$, where $n_{\mathrm{c}}$ is the critical electron density. For the front side $\mathrm{CD}$ targets, the pre-plasma region and a further $1 \mu \mathrm{m}$ of the maximum plasma density consists of a CD plasma followed by $6 \mu \mathrm{m}$ of aluminum plasma. For the rear side CD targets, the pre-plasma region and a further $6 \mu \mathrm{m}$ is aluminum plasma followed by $1 \mu \mathrm{m}$ of CD. On the rear side of both target types, there is a water $\left(\mathrm{H}_{2} \mathrm{O}\right)$ contamination layer of thickness, $L_{\mathrm{H}_{2} \mathrm{O}}$, which has the density of liquid water, $n_{\mathrm{H}}=6.64 \times 10^{22} \mathrm{~cm}^{-3}$ and $n_{\mathrm{O}}=3.32 \times 10^{22} \mathrm{~cm}^{-3}$.

The impact of the thickness of the water contaminant layer was found to be very influential on the acceleration of rear side deuterons. Other experiments have shown that the removal of the hydrogen rich contaminant layer from the target, either by thermal heating [33-35], using an argon-ion sputter gun [36,37] or using a secondary laser to ablate the surface [38, 39], allows more efficient acceleration of the bulk target material below. The simulation parameters to investigate the contaminant layer thickness were $I_{0}=3 \times 10^{19} \mathrm{~W} \mathrm{~cm}^{-2}, \tau_{\mathrm{FWHM}}=400 \mathrm{fs}$, $L_{\mathrm{Al}}=6 \mu \mathrm{m}, L_{\mathrm{CD}}=1 \mu \mathrm{m}$. Figure 1 shows the reduction in maximum deuteron energies, and increase in proton energies achieved, as the contaminant layer thicknesses is increased. This is to be expected as the strength of the electric field seen by the deuterons will be reduced by the shielding plasma generated by the contaminant layer. The protons in the contaminant layer gain most of the energy. An $L_{\mathrm{H}_{2} \mathrm{O}}=2 \mathrm{~nm}$ is chosen for the intensity variation simulations. There is a large scatter in the experimental data (figure $4(b)$ ), which could be partly due to varying contamination layer thicknesses over the $\mathrm{CD}$ layer as the acceleration has been shown to be so sensitive to $L_{\mathrm{H}_{2} \mathrm{O}}$. In contrast, because a density ramp was used to mimic the preplasma formation, the contaminant layer is expected to have little effect on the front side deuterons. 


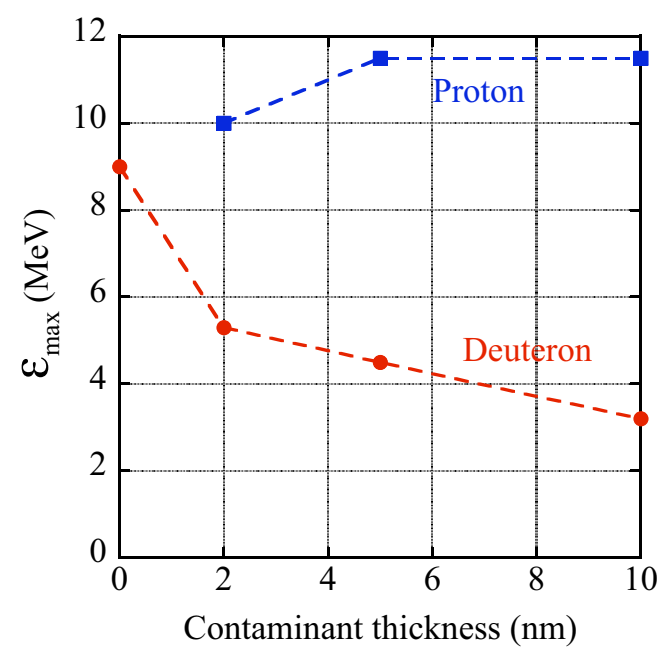

Figure 1. The maximum proton and deuteron energies achieved from simulations with different initial water contamination layer thicknesses, $L_{\mathrm{H}_{2} \mathrm{O}}$, over the rear CD layer. Simulation parameters, $I_{0}=3 \times 10^{19} \mathrm{~W} \mathrm{~cm}^{-2}, \tau_{\text {laser }}=400 \mathrm{fs}, L_{\mathrm{Al}}=6 \mu \mathrm{m}, L_{\mathrm{CD}}=1 \mu \mathrm{m}$.

It is worth noting that simulations were also performed with a layer on the rear side of the target of carbon, deuterons and protons homogeneously mixed. For this case, the deuteron acceleration is not suppressed by the contaminant layer and the deuterons gain comparable energy in comparable number to the protons. Having decided on $L_{\mathrm{H}_{2} \mathrm{O}}=2 \mathrm{~nm}$, the laser energy was varied to look at the dependence of the laser intensity on the ion acceleration.

\section{Results and discussion}

The dependence of maximum proton energy, $\mathcal{E}_{\text {max }, \mathrm{p}}$, and maximum deuteron energy, $\mathcal{E}_{\max , \mathrm{d}}$, on peak laser intensity for $6 \mu \mathrm{m}$ aluminum targets with different $\mathrm{CD}$ coatings is shown in figures $2(a)$ and $(b)$. Both $\mathcal{E}_{\text {max,p }}$ and $\mathcal{E}_{\text {max,d }}$ increase with laser intensity, up to around $1 \times 10^{19} \mathrm{~W} \mathrm{~cm}^{-2}$ and seem relatively insensitive as to whether the front or the rear side is coated with a CD layer. The results of the simulations are plotted alongside the experimental results in figure 2 and are shown using the open markers and are connected with a dashed line. $\mathcal{E}_{\text {max }, \mathrm{p}}$ from the simulations is largely unaffected by which side has the CD layer, which is in agreement with the experiment and possibly all these protons originate from the rear surface of the target. From the aluminum target, the experiment does not show such a sharp improvement in $\mathcal{E}_{\max , \mathrm{p}}$ with laser intensity, but this is likely to be because the pre-pulse is more severe at higher intensities and will cause a shock breakout on the rear of the target reducing the acceleration [29]. Hydrodynamic simulations presented by Lundh et al [30] suggest for the $6 \mu \mathrm{m}$ aluminum target this experiment is very close to shock breakout. Shot-to-shot variations in pre-pulse levels will contribute to scatter in the data by altering the plasma scale-length on the front surface, hence changing the energy absorption into fast electrons.

It might be expected that the protons and deuterons could potentially be accelerated to the same maximum energy in the rear sheath field. Figure 2(c) shows $\mathcal{E}_{\text {max,p }}$ against $\mathcal{E}_{\text {max,d }}$ and it is clear that the deuterons from the rear target surface are accelerated to far lower energies than the protons. Even when the rear surface is coated with the CD layer, a contamination layer of hydrocarbons will form over the top. Protons in the contamination layer will always see the 

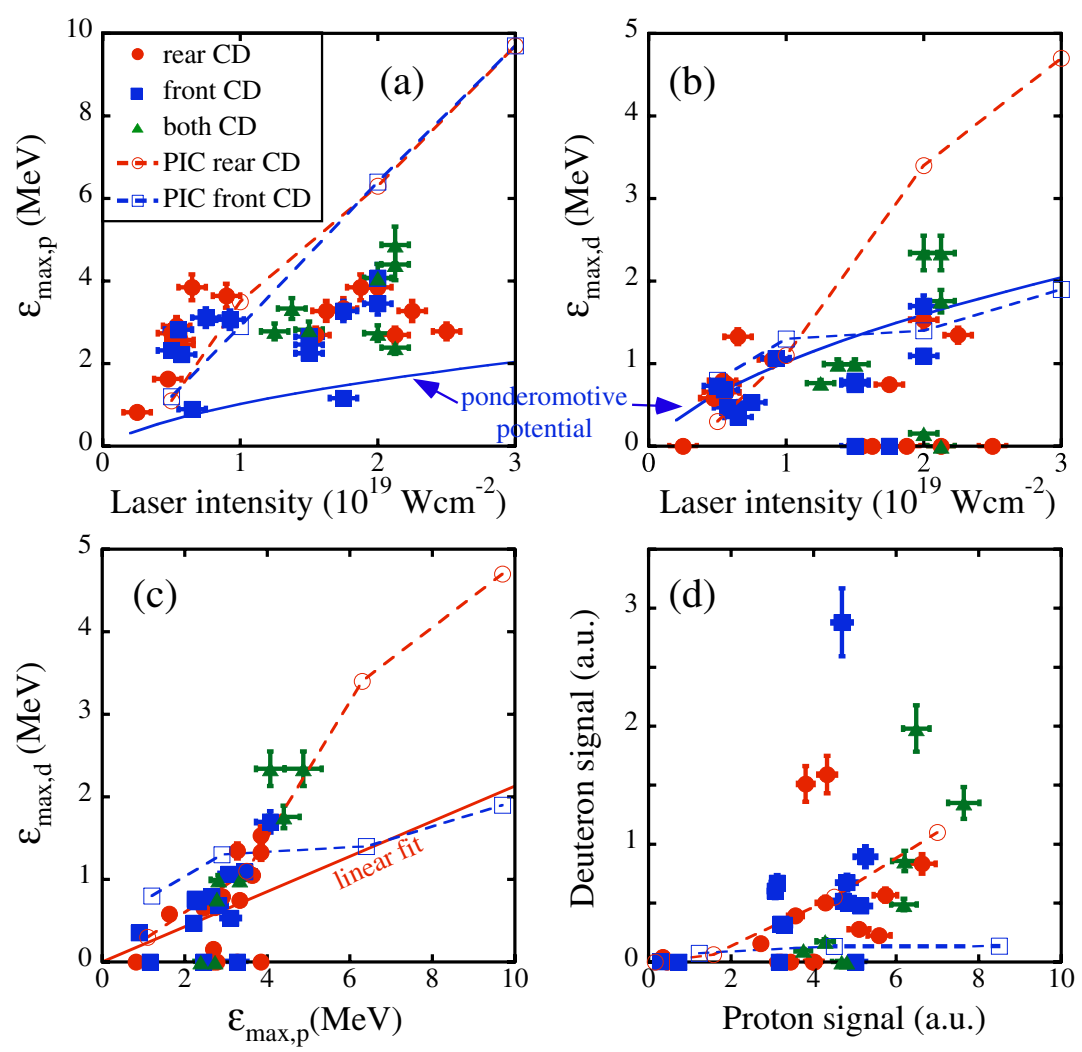

Figure 2. Experimental data for $6 \mu \mathrm{m}$ aluminum targets with different CD coatings. (a) $\mathcal{E}_{\text {max,p }}$ against laser intensity, $(b) \mathcal{E}_{\text {max,d }}$ against laser intensity, the solid blue line indicates the ponderomotive potential, $(c) \mathcal{E}_{\text {max,d }}$ against $\mathcal{E}_{\text {max,p }}$ and $(d)$ the proton signal against the deuteron signal. Open markers are for the PIC simulation results, which used $L_{\mathrm{H}_{2} \mathrm{O}}=2 \mathrm{~nm}$ for the rear $\mathrm{CD}$ simulations. In $(c)$ the solid line is a linear fit to the rear $\mathrm{CD}$ data, which gives the estimate $\mathcal{E}_{\text {max }, \mathrm{d}} \approx 0.2 \mathcal{E}_{\max , \mathrm{p}}$. The PIC simulation signals for $(d)$ are calculated using a $5^{\circ}$ cone and are normalized to overlay with the experimental data.

peak electric field because this outer proton front will shield the deuterons from the electric field and therefore the protons will be accelerated to the highest energy. Performing a linear fit to the rear $\mathrm{CD}$ coating data (shown in figure $2(c)$ as the solid red line) gives $\mathcal{E}_{\text {max,d }} \approx 0.2 \mathcal{E}_{\text {max, }}$.

There are various acceleration mechanisms from the front target surface [40]. At these experimental laser intensities, the ponderomotive potential is considered the main mechanism for front-surface ion acceleration. Other mechanisms, such as shock acceleration [13], dominate at somewhat higher laser intensities. The ponderomotive potential of the laser at the maximum intensity is $U_{\mathrm{p}}=m_{\mathrm{e}} c^{2}(\langle\gamma\rangle-1)$, where $m_{\mathrm{e}}$ is the mass of an electron, $c$ is the speed of light in a vacuum and $\langle\gamma\rangle$ is the time averaged relativistic Lorentz factor. For an ion of charge $Z$, the maximum energy an ion can gain at the front surface from the ponderomotive potential is $Z U_{\mathrm{p}}$ and this is plotted in figures $2(a)$ and $(b)$.

A deuteron accelerated from the front surface has to travel through the target and will therefore lose energy before it is measured. The minimum energy required for a deuteron to pass through $6 \mu \mathrm{m}$ of aluminum or $13 \mu \mathrm{m}$ of Mylar is $0.65 \mathrm{MeV}$ and $1.0 \mathrm{MeV}$, respectively, and these deuterons would emerge with very little remaining energy. This energy loss in the target will flatten the spectrum and reduce the maximum energy of the beam. Note that the PIC code 
does not account for collisional energy losses as the ion beam passes through the target, so a further downshift in the given PIC front $\mathrm{CD} \mathcal{E}_{\text {max }, \mathrm{d}}$ is expected. The energy loss inside the target has been accounted for in the given PIC data by transporting the deuterons originating from the front surface coated CD through the aluminum target. The Monte Carlo code used accounts for slowing down of the deuterons as well as their angular scattering [41]. Additional energy gain might be expected in the rear-surface sheath fields, as has been observed for positrons generated within a target [42]; however, this was not observed in the PIC simulations. Considering a thinner target, the energy loss would be less significant and therefore a far greater total deuteron signal is expected from the front surface. Also, the arrival time of ions from the front surface would be more prompt with the peak electric fields on the rear surface of a thin target.

The number of protons detected by the spectrometer considerably exceeds the number of deuterons. Figure $2(d)$ shows the relative proton signal to deuteron signal. The ion signal is calculated by summing the energy in each energy bin of the spectra to get a number proportional to an energy conversion efficiency of ions detected in the spectrometer. The total energy of protons and deuterons observed in the spectrometer increases with laser energy for all targets as does the ratio of proton-to-deuteron signal. The measured proton-to-deuteron signal is approximately $10: 1$ (figure $2(d)$ ), which is comparable to that observed in the rear CD simulations.

Figure 3 shows the experimental results from $13 \mu \mathrm{m}$ Mylar targets. Both front and rear side coated targets yield maximum proton energies comparable to that from the aluminum target (figure $3(a)$ ). $\mathcal{E}_{\max , \mathrm{p}}$ does not show a plateau with increasing intensity, suggesting rear side shock breakout is not occurring for the $13 \mu \mathrm{m}$ Mylar targets. The deuterons from the front and rear surfaces are observed to have fairly comparable $\mathcal{E}_{\text {max,d }}$ (figure $3(b)$ ). However, it is clear from the proton-to-deuteron signal ratio (figure $3(d)$ ) that there were far fewer measured deuterons from the front $\mathrm{CD}$ coated targets.

The front surface coated CD, particularly at lower intensities, exhibits large scatter in both $\mathcal{E}_{\text {max,d }}$ and deuteron signal, with some shots being null shots where no deuterons were detected. The data scatter and null shots are explained by the larger spectral shift in traveling through the target, which will be more pronounced for the thicker $13 \mu \mathrm{m}$ Mylar target compared with the thinner $6 \mu \mathrm{m}$ aluminum. The effects will be particularly severe at lower intensities where deuterons near the minimum energy threshold are expected. Large data scatter and null shots for the rear-surface coated $\mathrm{CD}$ are due to variations in the contaminants residing on the rear target surface. The contaminant layer thickness cannot be precisely controlled and its impact on both $\mathcal{E}_{\text {max }, \mathrm{d}}$ and ion signal is significant, producing the observed large scatter in the data. The simulations suggest that a contaminant layer thicker than $10 \mathrm{~nm}$ may produce zero or undetectable maximum deuteron energies and signal.

Figures 4 and 5 show the $(a)$ proton and $(b)$ deuteron spectra for three shots $\left(I_{0} \approx\right.$ $2 \times 10^{19} \mathrm{~W} \mathrm{~cm}^{-2}$ ) onto $6 \mu \mathrm{m}$ aluminum and $13 \mu \mathrm{m}$ Mylar targets, respectively. The proton spectra are very similar, particularly for the $6 \mu \mathrm{m}$ aluminum, even though the targets had different $C D$ coatings, one rear, one front and one both sides. More surprising is that the deuteron spectra are very comparable in number and energy. The deuteron spectra typically display bumps and peaks in the spectra $[35,43,44]$, which would not be well described by a temperature. Target composition is likely to be influencing the ion spectra: a relatively low proton density can give rise to peaks in the energy spectrum of ions accelerated from the rear side of the target due to the influence of different ion species on the sheath field [44]. Deuterons accelerated from the front surface have to first travel through the target to emerge from the rear side and the energy loss for lower energy ions will be more pronounced than for higher energy, leading to a shift in the spectra and may lead to a low-energy cut-off. Another factor to consider is the angular divergence of the accelerated beams, because the Thomson parabola 

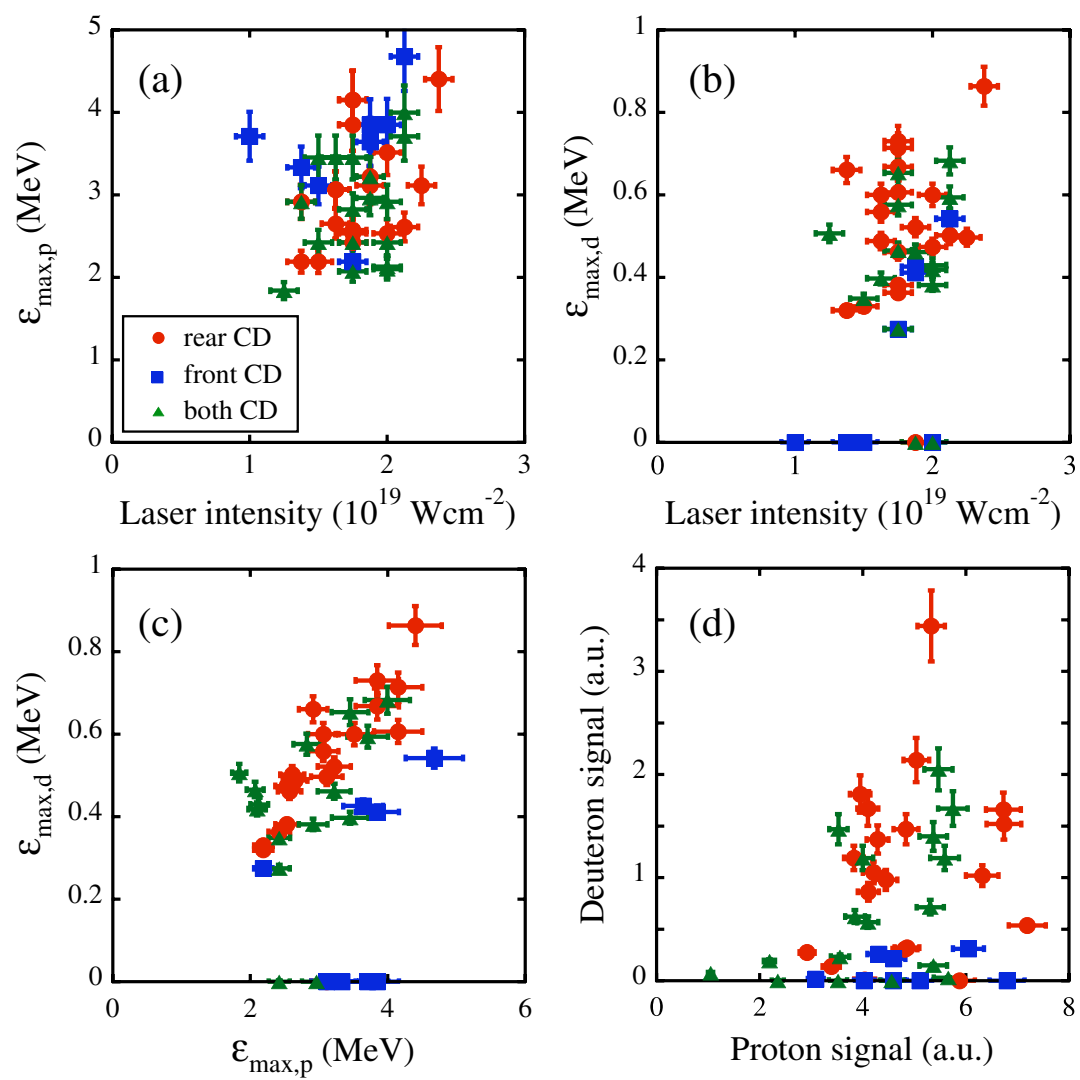

Figure 3. Experimental data for $13 \mu \mathrm{m}$ Mylar targets with different CD coatings. (a) $\mathcal{E}_{\text {max,p }}$ against laser intensity. (b) $\mathcal{E}_{\text {max,d }}$ against laser intensity. (c) $\mathcal{E}_{\text {max,d }}$ against $\mathcal{E}_{\text {max,p. }}$ (d) proton signal against deuteron signal.

spectrometer only measures the spectrum from a small solid angle, $\Omega \approx 2 \times 10^{-7} \mathrm{sr}$. The angular divergence of the proton beam from uncoated targets was measured to be $\approx 30^{\circ}$ (half beam, full width) at $1 \mathrm{MeV}$ and becomes smaller with higher energies. It is not possible to measure the deuteron angular divergence in this way as it cannot be distinguished from the proton signal.

The ion spectra seen in the $2 \times 10^{19} \mathrm{~W} \mathrm{~cm}^{-2}$ simulation are shown in figure $4(c)$. The protons show higher maximum energies than the deuterons, which agrees well with the experimental data, but exhibits a low-energy cut-off. This low-energy proton cut-off is attributed to the fact that the proton source layer is very thin, $2 \mathrm{~nm}$, which has been shown to produce quasi-monoenergetic features [35]. During the simulations, the proton layer becomes depleted and with no further protons in the CD layer behind, the spectrum shows a low-energy cut-off. However, the feature is not seen in the experiment because it is expected that the rear CD layer will contain a significant proportion of hydrogen and would provide the low-energy part of the proton spectrum. Also, protons accelerated from the front surface (not modeled in the simulations) would downshift in energy as they pass through the target and then can contribute to the low-energy part of the spectrum observed in the experiment.

Another expectation may be that the absorption of the laser into fast electrons differs depending on what target material the laser interacts with and what pre-plasma scale length 

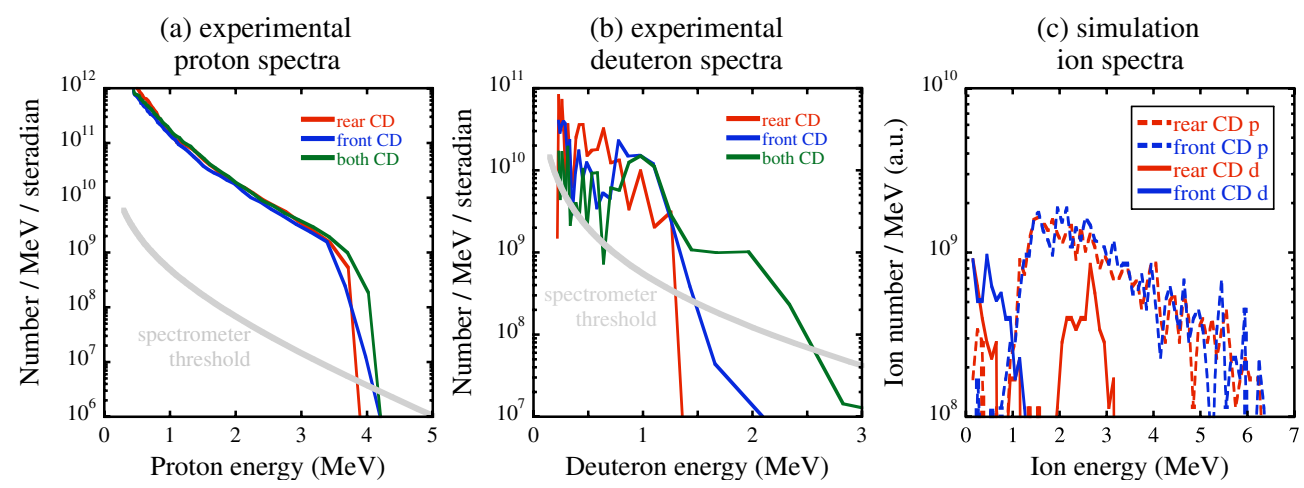

Figure 4. Comparison of proton and deuteron spectra for $6 \mu \mathrm{m}$ aluminum with front, rear or both sides coated targets: experimental spectra are given in $(a)$ and $(b)$ for protons and deuterons, respectively, for $I_{0} \approx 2 \times 10^{19} \mathrm{~W} \mathrm{~cm}^{-2}$ and $(c)$ is PIC simulation ion spectra within a $5^{\circ}$ cone around the target normal direction for $I_{0}=2 \times 10^{19} \mathrm{~W} \mathrm{~cm}^{-2}$ (front accelerated deuterons are corrected for energy loss through the target).
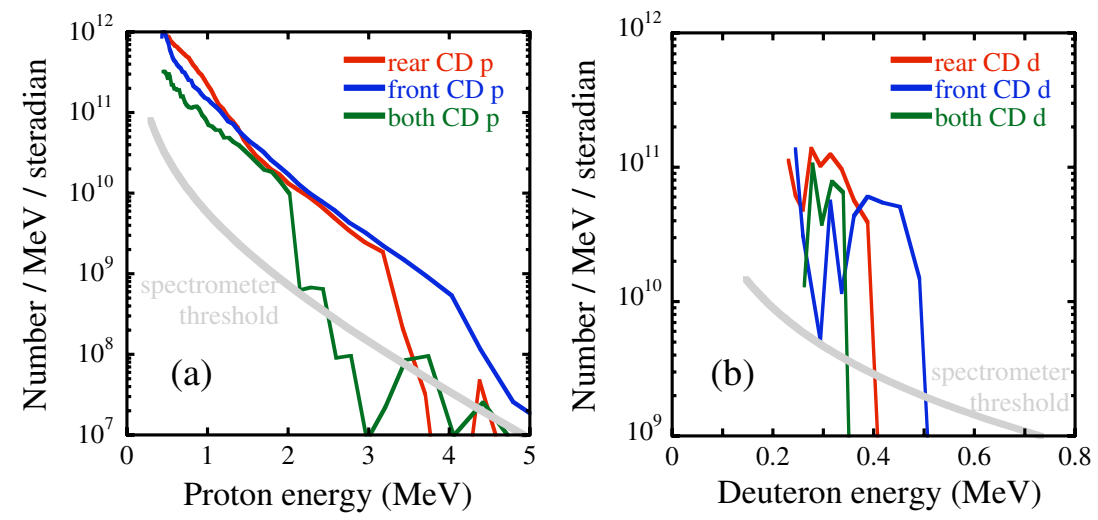

Figure 5. Comparison of experimental $(a)$ proton and $(b)$ deuteron spectra for $13 \mu \mathrm{m}$ Mylar with front, rear or both sides coated targets from $I_{0} \approx 2 \times 10^{19} \mathrm{~W} \mathrm{~cm}^{-2}$ shots.

is present [45]. At lower intensities, $\sim 10^{18} \mathrm{~W} \mathrm{~cm}^{-2}$, the target material has been found to be influential on front side proton acceleration [46]. However, the proton and deuteron maximum energies and spectra are similar whether the front surface is aluminum or deuterated plastic, suggesting that there is little influence in this experiment.

\section{Summary}

In conclusion, this experiment observed high-resolution light-ion spectra in the rear target normal direction and show that under certain conditions there can be a significant contribution from a front side acceleration mechanism. For these laser and target parameters, similar maximum ion energies can be achieved in the target normal rear direction from acceleration at both the front and rear of the target. The importance of the thickness of any contamination layer over the target material on the acceleration of the bulk target material in rear side acceleration is observed from PIC simulations and this is an interesting subject for future study. To achieve 
maximum acceleration for rear side originating deuterons for applications such as neutron production, removal of the contamination layer will be necessary, even though deuterons are the lightest ions after protons.

\section{Acknowledgments}

This work was supported by the Defense Threat Reduction Agency (DTRA) and the Naval Research Laboratory (NRL).

\section{References}

[1] Clark E L et al 2000 Phys. Rev. Lett. 84670

[2] Maksimchuk A, Gu S, Flippo K, Umstadter D and Bychenkov V Yu 2000 Phys. Rev. Lett. 844108

[3] Snavely R A et al 2000 Phys. Rev. Lett. 852945

[4] Bulanov S V, Esirkepov T Z, Khoroshkov V S, Kunetsov A V and Pegoraro F 2002 Phys. Lett. A 299240

[5] Fourkal E, Shahine B, Ding M, Li J, Tajima T and Ma C 2002 Med. Phys. 292788

[6] Malka V et al 2004 Med. Phys. 311587

[7] Borghesi M, Campbell D H, Schiavi A, Willi O, MacKinnon A J, Hicks D, Patel P, Gizzi L A, Galimberti M and Clarke R J 2002 Laser Part. Beams 20269

[8] Roth M et al 2001 Phys. Rev. Lett. 86436

[9] Bychenkov V Yu, Rozmus W, Maksimchuk A, Umstadter D and Capjack C E 2001 Plasma Phys. Rep. 271017

[10] Wilks S C, Langdon A B, Cowan T E, Roth M, Singh M, Hatchett S, Key M H, Pennington D, MacKinnon A and Snavely R A 2001 Phys. Plasmas 8542

[11] Pukhov A 2001 Phys. Rev. Lett. 863562

[12] Badziak J, Głowacz S, Jabłoński S, Parys P, Wołowski J, Hora H, Krása J, Láska L and Rohlena K 2004 Plasma Phys. Control. Fusion 46 B541

[13] Silva L O, Marti M, Davies J R, Fonseca R A, Ren C, Tsung F S and Mori W B 2004 Phys. Rev. Lett. 92015002

[14] Habara H et al 2004 Phys. Rev. E 7046414

[15] Karsch S, Düsterer S, Schwoerer H, Ewald F, Habs D, Hegelich M, Pretzler G, Pukhov A, Witte K and Sauerbray R 2003 Phys. Rev. Lett. 91015001

[16] Esirkepov T, Borghesi M, Bulanov S V, Mourou G and Tajima T 2004 Phys. Rev. Lett. 92175003

[17] Robinson A P L, Zepf M, Kar S, Evans R G and Bellei C 2008 New J. Phys. 10013021

[18] Macchi A, Veghini S and Pegoraro F 2009 Phys. Rev. Lett. 103085003

[19] Bulanov S S et al 2008 Phys. Rev. E 78026412

[20] Henig A et al 2009 Phys. Rev. Lett. 103245003

[21] Fuchs J et al 2005 Phys. Rev. Lett. 94045004

[22] Fuchs J et al 2007 Phys. Plasma 14053105

[23] Izumi N et al 2002 Phys. Rev. E 65036413

[24] Petrov G M and Davis J 2008 Plasma Phys. Control. Fusion 50015004

[25] Willingale L, Petrov G M, Maksimchuk A, Freeman R R, Matsuoka T, Murphy C D, Ovchinnikov V M, Van Woerkom L and Krushelnick K 2009 in preparation

[26] McKenna P et al 2008 Laser Part. Beams 26591

[27] Glinec Y, Genoud G, Lundh O, Persson A and Wahlström C-G 2008 Appl. Phys. B 93317

[28] Nuter R, Gremillet L, Combis P, Drouin M, Ledebvre E, Flacco A and Malka V 2008 J. Appl. Phys. 104103307

[29] Kaluza M, Schreiber J, Santala M K I, Tsakiris G D, Eidmann K, Meyer-ter-Vehn J and Witte K J 2004 Phys. Rev. Lett. 93045003

[30] Lundh O, Lindau F, Persson A, Walström C-G, McKenna P and Batani D 2007 Phys. Rev. E 76026404

[31] Harres K et al 2008 Rev. Sci. Instrum. 79093306

[32] Petrov G M and Davis J 2008 Comput. Phys. Commun. 179868

[33] Hegelich M et al 2002 Phys. Rev. Lett. 89085002

[34] McKenna P et al 2004 Phys. Rev. E 70036405

[35] Hegelich B M, Albright B J, Cobble J, Flippo K, Letzring S, Paffett M, Ruhl H, Schreiber J, Schulze R K and Fernández J C 2006 Nature 439441

[36] Allen M, Patel P K, Mackinnon A, Price D, Wilks S and Morse E 2004 Phys. Rev. Lett. 93265004

[37] Offermann D T et al 2009 Phys. Plasma 16093113

[38] Fernández J C et al 2005 Laser Part. Beams 23 267-73 
[39] Pfotenhauer S M et al 2008 New J. Phys. 10033034

[40] Borghesi M, Fuchs J, Bulanov S V, MacKinnon A J, Patel P K and Roth M 2006 Fusion Sci. Technol. 49412

[41] Davis J and Petrov G M 2008 Plasma Phys. Control. Fusion 50065016

[42] Chen H, Wilks S C, Bonlie J D, Liang E P, Myatt J, Price D F, Meyerhofer D D and Beiersdorfer P 2009 Phys. Rev. Lett. 102105001

[43] Schwoerer H, Pfotenhauer S, Jäckel O, Amthor K-U, Liesfeld B, Ziegler W, Sauerbrey R, Ledingham K W D and Esirkepov T 2006 Nature 439445

[44] Robinson A P L, Bell A R and Kingham R J 2006 Phys. Rev. Lett. 96035005

[45] Lefebvre E and Bonnaud G 1997 Phys. Rev. E 55 1001-14

[46] Lee K, Park S H, Cha Y-H, Lee J Y, Lee Y W, Yea K-H and Jeong Y U 2008 Phys. Rev. E 78056403 